\title{
TRASTORNOS DEL SUEÑO Y TRASTORNOS DE LA CONDUCTA ALIMENTARIA. RELAJACIÓN CON RISOTERAPIA. ESTUDIO PILOTO
}

\section{MARÍA ROSARIO VALERA FERNÁNDEZ1 ${ }^{1}$, JORGE COLETAS JUANICO², MIGUEL ÁNGEL RAMÍREZ SÁNCHEZ-CARNERERO', ANNA GRACIA ARNAU1, ANA BELÉN VALERA FERNÁNDEZ ${ }^{3}$ Y MARÍA JOSÉ MARÍN ARTERO ${ }^{3}$}

${ }^{1}$ Profesional de enfermería especialista en Salud Mental del Institut de Neuropsiquiatria i Addiccions (INAD). Consorci Mar Parc de Salut de Barcelona.

2Profesional de enfermería especialista en Salud Mental. Unidad de Crisis del INAD. Consorci Mar Parc de Salut de Barcelona.

${ }^{3}$ Profesional de enfermería de la Unidad de Hospitalización de Subagudos del INAD. Consorci Mar Parc de Salut de Barcelona.

\section{RESUMEN}

Introducción: Los pacientes con trastornos de la conducta alimentaria presentan dificultades en varios ámbitos de su vida, entre ellos el sueño. El insomnio tiene consecuencias, como la somnolencia diurna, un peor aspecto físico, menor rendimiento y empeoramiento del estado de ánimo. La risoterapia es una técnica de relajación que mejora el estado físico y psicológico a través de la risa.

Método: Estudio de investigación metodológica analítica de corte longitudinal retrospectivo. Se utilizó el Cuestionario de Pittsburg sobre calidad de sueño y se midió el nivel de ansiedad antes y después de la técnica de risoterapia mediante la Escala análoga visual de Abu-Saad.

Resultados y discusión: Insomnio de conciliación, 83,3\%; insomnio de mantenimiento, 66,6\%, y presencia de pesadillas, $66,6 \%$, lo que en conjunto lleva a una sensación subjetiva de mala calidad de sueño en el 83,3\%. Los problemas de sueño mejoran con la práctica de risoterapia.

Palabras clave: trastornos de la conducta alimentaria, trastornos del sueño, relajación, risoterapia.

Correspondencia: M. R. Valera.

Correo electrónico: charo-valera@hotmail.com 


\section{INTRODUCCIÓN}

Los trastornos de la conducta alimentaria (TCA) -anorexia nerviosa, bulimia nerviosa y cuadros afines 0 no especificados- son uno de los problemas en salud mental que más preocupan a los profesionales, y para algunos de ellos han llegado a tener tintes de alarmante fenómeno epidémico. Han alcanzado una especial relevancia en las tres últimas décadas, tanto por su creciente incidencia como por la gravedad de la sintomatología asociada y la resistencia al tratamiento; hoy día constituyen la tercera enfermedad crónica entre la población femenina adolescente y juvenil en las sociedades desarrolladas y occidentalizadas ${ }^{1}$. La opinión más generalizada en lo referente a la etiología de los trastornos alimentarios es que tienen un origen multicausal, entre los que la preocupación por la figura y el seguimiento de dietas de adelgazamiento se consideran conductas de riesgo².

En el marco de los trastornos alimentarios se define la imagen corporal como la representación mental de diferentes aspectos de la apariencia física formada por tres componentes: el perceptivo, el cognitivo-afectivo y el conductual. La alteración de la imagen corporal supone un desequilibrio o perturbación en sus componentes ${ }^{3}$, y la alteración de la imagen corporal tiene una participación causal en los trastornos alimentarios ${ }^{4}$, aspecto más preocupante todavía si tenemos en cuenta que durante la adolescencia se produce un incremento de la insatisfacción corporal.

En investigaciones anteriores se ha comprobado que las personas que sufren anorexia en la adolescencia suelen presentar las siguientes características ${ }^{5}$ :

- Alteraciones de la imagen corporal.

- Hiperactividad, como actividad física alta y extenuante.

- Restricción de la ingesta de alimentos y/o su eliminación mediante purgas y ejercicio excesivo.

- Una pérdida de peso que conduce a la desnutrición, que se mide a través de dos parámetros: el mantenimiento de un peso por debajo del $85 \%$ del establecido para su edad y altura, y un índice de masa corporal igual o inferior a 17,5.

- Alteraciones en el ciclo menstrual, con amenorrea.

- Negación y control del hambre; aunque la tienen, lo niegan.

- Perfeccionismo, imponiéndose unas metas difíciles de conseguir, lo que les lleva a tener una gran minuciosidad en la ejecución, motivo por el cual mantienen una sensación de fracaso permanente.

- Obsesiones y control, llegando a tener una degadez que no es sostenible biológicamente; por ello el cuerpo lucha por incrementar el deseo de comer y los pensamientos relacionados con la comida. Al final, puede convertirse en un trastorno obsesivo grave.

- Miedo a la madurez, pues la restricción de alimento hace que el paciente detenga su crecimiento y permanezca con un cuerpo de niño/a, sin ser capaz de hacer el paso a la adolescencia.

- Exaltación y sensación de fuerza, que se produce cuando valora que ha cubierto sus objetivos: delgadez, control de la comida y evitar su existencia.

- Irritabilidad y fluctuaciones bruscas del estado de ánimo, pues el acto de comer le provoca culpabilidad, ya que no ha sido capaz de controlarse.

- Desequilibrio en el estilo de vida, dado que su forma de actuar la extrapola al terreno académico o profesional.

Los TCA son patologías en las que el curso de la enfermedad hace recomendables períodos de tratamiento intensivo ante descompensaciones médicas y psiquiátricas que hacen peligrar la evolución del paciente.

Los ingresos hospitalarios no son la única opción de tratamiento intensivo. Los programas de hospitalización diurna u hospitales de día (HD) para TCA han adquirido una importancia creciente en la atención de estas patologías. A través de una atención 
escalonada se proporciona la necesaria asistencia al paciente y su familia.

Los programas de HD como cuidados intermedios son un recurso necesario según esta filosofía. Están situados a medio camino entre la hospitalización a tiempo completo y el tratamiento ambulatorio. Los HD deben contar con camas hospitalarias especializadas o tener acceso a ellas, y contar con un cuidadoso programa de posalta y seguimiento ${ }^{6}$. Fueron creados para evitar o disminuir el tiempo de ingreso, por su menor coste económico y posibles ventajas terapéuticas ${ }^{7}$.

Los pacientes con TCA tienen asociadas unas características emocionales y unas creencias particulares sobre la comida y la imagen corporal, lo que ocasiona dificultades en varios ámbitos de su vida diaria, como la desnutrición, deshidratación, empeoramiento y/o pérdida de relaciones sociales, sintomatología depresiva y/o problemas del sueño.

Debido a la cantidad de problemas que acarrea esta enfermedad, una intervención adecuada es crucial para obtener una correcta salud. En este estudio nos centramos en los trastornos del sueño debido a su alta incidencia en esta población.

Entre los trastornos del sueño existentes encontramos el síndrome de las piernas inquietas, apneas del sueño, insomnio, enuresis, terrores nocturnos, etc. Los artículos científicos sobre sueño y TCA mencionan el insomnio como problema más frecuente en personas que sufren esta enfermedad ${ }^{8,9}$.

El hecho de no dormir bien tiene una serie de consecuencias para la persona que lo sufre, como son la somnolencia diurna, un peor aspecto físico (impresión de cansancio, desaliño y presencia de ojeras), menor rendimiento (tanto académico como en actividades y/o relaciones personales) y empeoramiento del estado de ánimo, con mayor presencia de sintomatología depresiva y ansiosa (irritabilidad, nerviosismo, cambios bruscos de humor, etc. ${ }^{10}$.

Una de las medidas de mejora de los problemas del sueño son las técnicas de relajación, ya que estas regulan la respiración (lo que ayuda a una mayor oxigenación de las células y el cerebro), reducen la tensión muscular (lo que contribuye a mejorar algunas condiciones de dolor), reducen los niveles de algunas sustancias fisiológicas implicadas en la ansiedad (adrenalina, noradrenalina, lactato sanguíneo), contribuyen a lograr un estado de descanso profundo, conducen a un estado de relajación y claridad mental que tiene un impacto positivo a la hora de poner en práctica estrategias de resolución de problemas, y también contribuyen a generar pensamientos positivos y creencias positivas, lo que ayuda a mejorar las estrategias de afrontamiento de la persona, ya que puede incrementar la sensación de seguridad y autoconfianza11,12. Por este motivo, consideramos en nuestra hipótesis que los trastornos del sueño presentes en usuarios con TCA se verían mejorados con la utilización periódica de técnicas de relajación que redujeran el estado de ansiedad basal de los individuos.

Entre las técnicas de relajación más utilizadas encontramos: respiración abdominal (inspirar y espirar profundamente), relajación de Jacobson (que consiste en la contracción/relajación de varios grupos musculares de forma progresiva), relajación pasiva (esta técnica no utiliza ejercicios de tensión; puede resultar indicada cuando los sujetos presentan dificultades o incapacidad orgánica para tensar los músculos o relajarlos una vez tensados), relajación autógena (que consiste, básicamente, en una serie de frases elaboradas con el fin de inducir en el sujeto estados de relajación a través de autosugestiones sobre: sensaciones de pesadez y calor en sus extremidades, regulación de los latidos de su corazón, sensaciones de tranquilidad y confianza en sí mismo y concentración pasiva en su respiración) ${ }^{13,14}$ y otras técnicas alternativas, que actualmente están en auge, como son la musicoterapia, la aromaterapia, la masoterapia, el mindfulness, la risoterapia, etc.

La risoterapia es la técnica empleada para la realización de este estudio. Se considera una terapia destinada a mejorar el estado físico y psicológico a través de la risa; y es que cuando una persona ríe, 
además de mejorar su estado de ánimo y mejorar su estado psicológico o de estrés, con la risa también se genera una sustancia benéfica para su organismo de cara a diferentes patologías y/o enfermedades: las endorfinas ${ }^{15}$.

Esta terapia se realiza comúnmente en sesiones/ talleres donde se educa a los participantes en todos los conceptos de la risa, sus tipos, ejercicios de relajación, cómo provocar la risa... y sobre todo la puesta en práctica para fomentar toda la positividad de los participantes y hacer pasar un muy buen rato de diversión, risas y beneficio psicológico y físico al mismo tiempo ${ }^{16}$.

Para conseguir el objetivo propuesto, se utilizan técnicas que ayudan a liberar las tensiones del cuerpo y así poder llegar a la carcajada. Entre ellas, la expresión corporal, el juego, la danza, ejercicios de respiración, masajes, técnicas para reír de manera natural, sana, que salga del corazón, del vientre, de un modo simple.

Las ventajas de la práctica de la risoterapia son muchas. Entre ellas destacan: la disminución de la percepción del dolor; rebaja el nivel de cortisol, al combatir el estrés y el insomnio; mejora la función pulmonar en pacientes asmáticos; aumenta el funcionamiento del sistema inmunitario; reduce la ansiedad en el preoperatorio, sobre todo en los niños; limpia los ojos y despeja de mucosidad la nariz y los oídos; ayuda a solucionar algunos problemas dermatológicos; combate problemas cardiovasculares; mejora los cuadros de estreñimiento; relaja las contracturas en la espalda, etc. ${ }^{17,18}$ (tabla 1).

\section{MATERIAL Y MÉTODO}

El HD de TCA del Hospital Universitario Clínic de Barcelona es un dispositivo ambulatorio que atiende pacientes con TCA que no precisan ingreso, pero sí abordaje intensivo de 14 a 21 horas y personalizado, programas de desensibilización y realización de grupos específicos, desde un enfoque multidisciplinar. Dispone de un máximo de 20 plazas y una estancia media de ingreso de entre 3 y 4 meses. Se

Tabla 1. Efectos de la terapia

- Vibran las cuerdas vocales

- Se produce lagrimeo

- Se abre la glotis

- 15 músculos de la cara se contraen y relajan en armonía

- Aumenta la frecuencia cardíaca y la respiración

- Se mueven diversos músculos, entre ellos abdominales y torácicos

- Se agitan brazos y piernas

- Se queman calorías

- Nos puede producir hasta cansancio físico, dolor de estómago y agujetas

- Es un anestésico contra el dolor

- Estimula la circulación de la sangre

- Se liberan endorfinas y neurohormonas

- Se segrega adrenalina

- Aumentan las inmunoglobinas

- Aumentan los linfocitos 
accede al servicio previa derivación desde urgencias, médico de familia, consultas externas o el centro de salud mental infanto-juvenil. La edad máxima de ingreso es de 18 años.

Se trata de un estudio de investigación metodológica analítica de corte longitudinal retrospectivo.

Como se ha mencionado anteriormente, se realizan grupos de trabajo específicos de unos 7 usuarios, que se renuevan cada 2 meses aproximadamente. Se trata de grupos teórico-prácticos que ayudan a los usuarios a reconocer sus errores cognitivos, causantes de gran parte del malestar existente, y a adquirir un sistema de pensamiento racional que les permita interpretar la realidad de manera objetiva, llegando a moderar la intensidad de sus emociones negativas.

Para este estudio piloto se selecciona una muestra de 6 usuarios del HD; 5 son chicas y uno, chico, con edades comprendidas entre los 14 y 16 años. La estancia previa de ingreso de la muestra es aproximadamente de 1 mes.

En este estudio se pretende comprobar si mejora el nivel de ansiedad basal, y en consecuencia mejora la calidad del sueño y disminuye el insomnio, mediante la práctica de talleres de risoterapia.

Para la valoración del estudio se utilizan dos cuestionarios:

1. Cuestionario de Pittsburg de calidad de sueño: es un cuestionario autoadministrado. Consta de 19 ítems autoevaluados por el paciente y 5 cues- tiones evaluadas por el compañero/a de cama. Las cinco últimas cuestiones se utilizan como información clínica, pero no contribuyen a la puntuación total. Los 19 ítems analizan los diferentes factores determinantes de la calidad del sueño, que se agrupan en 7 componentes: calidad del sueño, latencia del sueño, duración del sueño, eficiencia del sueño, alteraciones del sueño, uso de medicación para dormir y disfunción diurna ${ }^{19}$. Este cuestionario se pasó a la población diana una semana antes de las sesiones y una semana después de acabarlas para comprobar el grado de mejoría del sueño, una vez realizadas las sesiones.

2. Escala análoga visual de la ansiedad de Abu-Saad. Se trata de una escala gráfica de dos caras, con expresiones faciales opuestas unidas por una línea sin referencias numéricas, en la que se pide al usuario que señale el punto que mejor define la ansiedad presente. Esta escala también se evalúa antes y después de cada sesión (fig. 1).

El estudio se divide en dos sesiones, una vez a la semana, de 2 horas de duración. En la primera sesión, mediante una presentación de PowerPoint se informa a los participantes de los problemas del sueño detectados en la muestra y las consecuencias de estos, desde una perspectiva bidireccional. Se realiza educación sanitaria sobre hábitos de higiene del sueño y se les muestran las técnicas de relajación más utilizadas. Para finalizar la sesión, se pone en práctica una combinación de varias técnicas (res-

Figura 1. Escala análoga visual de la ansiedad de Abu-Saad
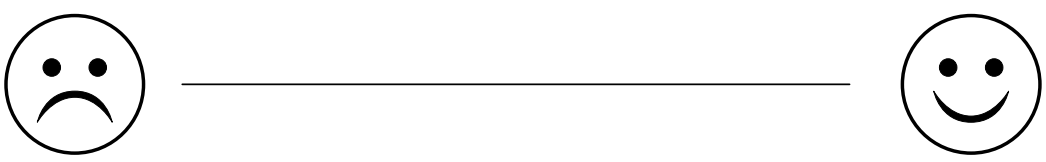
piración abdominal, relajación autógena y visualización) adaptada a usuarios que padecen un TCA.

En la segunda sesión nos centramos en el taller de risoterapia, aportando información en primer lugar sobre los beneficios de la risa. Posteriormente, se realizan diferentes dinámicas grupales de risoterapia, entre ellas: rueda de muecas y risas, juego del globo, explorar la risa, la granja de los animales, "cadáver exquisito», técnica de las cosquillas, duelo de risas, etc.

\section{RESULTADOS Y DISCUSIÓN}

Tras la realización del estudio, se ha comprobado que el nivel de ansiedad se reduce y mejora la calidad del sueño tras practicar una técnica de relajación, como en este caso es la risoterapia.

Los resultados obtenidos tras el estudio de los trastornos del sueño más frecuentes en la muestra piloto, mediante la utilización del Cuestionario de
Pittsburg, han sido en primer lugar el insomnio de conciliación con un 83,3\% de los casos, seguido del insomnio de mantenimiento, cuya representación es del 66,6\% y la presencia de pesadillas o malos sueños relacionados principalmente con la enfermedad, con otro $66,6 \%$ de los participantes. Todo ello lleva a una sensación subjetiva de mala calidad de sueño en el 83,3\% de la población de estudio (fig. 2), por lo que los resultados obtenidos son semejantes a los de otros estudios científicos, como los de Walsh et al. ${ }^{20}$ y Delvene et al..21, quienes documentaron en pacientes anoréxicas una menor eficiencia del sueño, algunos problemas de insomnio intermedio y una menor duración del sueño.

Igualmente, sobre el nivel de ansiedad pre- y postsesión, valorado con la Escala análoga visual de Abu-Saad, se obtiene un nivel previo de 8,3 puntos, y después de la dinámica grupal, de 5,1, lo que sugiere la mejora del nivel de ansiedad basal detectado en usuarios adolescentes con un TCA.

Figura 2. Trastornos del sueño detectados en usuarias con trastornos de la conducta alimentaria

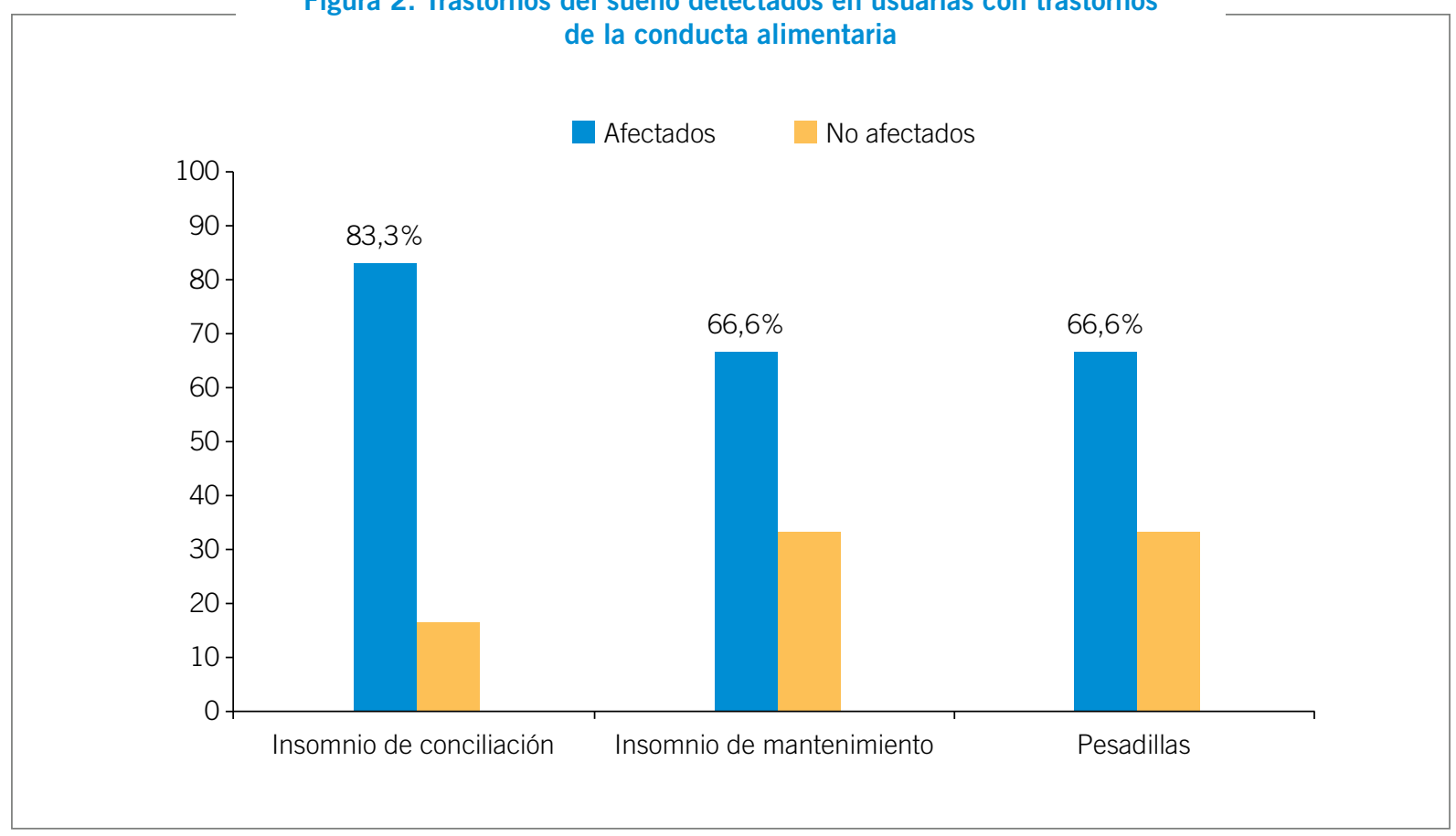


Los resultados indican que los problemas subjetivos graves de dificultad para mantener el sueño y sensación de no haber tenido un sueño reparador se asocian con problemas de imagen corporal e intentos recurrentes de perder peso entre las mujeres jóvenes. Nuestros resultados acerca de la frecuencia de problemas de sueño y de TCA están en la línea de investigaciones anteriores.

También es cierto que la gente joven tiende a dormir menos de lo que necesita; un estudio de Strauch y Meier 22 mostró una discrepancia entre la duración del sueño normal y la deseada de 1,7 horas en los días entre semana y un deseo de dormir más entre un $54 \%$ y un $75 \%$ de los sujetos. En nuestro estudio, el 83,3\% refirió un déficit subjetivo de tiempo de sueño.

En relación con que la dificultad para mantener el sueño sea el insomnio más típico de los individuos con TCA, un estudio de laboratorio de Maes et al. ${ }^{23}$ es relevante para la discusión. El estudio mostró que la dificultad para mantener el sueño en sujetos deprimidos se asociaba a signos indirectos de aumento del recambio de catecolaminas, ansiedad somática e hipocondriasis, «un estado de hiperalerta».

La risoterapia ha demostrado ser una técnica alternativa de relajación tan eficaz como las medidas más tradicionales, aportando además de los beneficios propios de la relajación, otras cualidades extras, como la mayor unión de los miembros del grupo, el autoconocimiento a través de la risa, la mejora de la autoestima, etc.

Como limitación del estudio encontramos la escasa muestra disponible. En un futuro es imprescindible verificar los resultados obtenidos con una muestra más representativa de usuarios con TCA.

\section{BIBLIOGRAFÍA}

1. Peláez Fernández MA, Labrador Encinas FJ, Raich Escursell RM. Epidemiología de los trastornos de la conducta alimentaria en España: revisión y estado de la cuestión. Cuad Med Psicosom Psiquiatr Enlace. 2004;71/72:33

2. Toro J. La epidemiología de los trastornos de la conducta alimentaria. Med Clin (Barc). 2000;114:543-4.

3. Thompson JK. Body image disturbance: assessment and treatment. Elmosford NY: Pergamon Press; 1990.

4. Baile JI, Guillén F, Garrido E. Insatisfacción corporal con adolescentes medida con el Body Shape Questionnaire (BSQ): efecto del anonimato, el sexo y la edad. Int J Clin Health Psychol. 2002;2:439-50.

5. Garrote Rojas D. Análisis de la incidencia de los trastornos de la conducta alimentaria (TCA) en los factores de personalidad de los estudiantes de educación secundaria. Granada: Publicaciones de la Universidad; 2009.

6. Morandé G. Claves para la atención de los trastornos de la conducta alimentaria (TCA). Del tratamiento a la prevención. Vigo: Universidad de Vigo; 2000.

7. Turón V. Hospitales de día para los trastornos de la conducta alimentaria. En: Rojo Moreno L, Cava G, editores. Anorexia nerviosa. Barcelona: Ariel Ciencias Médicas; 2003. p. 395-410.

8. De la Torre Luque A, Miró Morales E, Martínez Narváez MP. Calidad subjetiva de sueño en pacientes con trastornos de la conducta alimentaria. Apunt Psicol. 2013; 31(1):11-20.

9. Seigel K, Broman JE, Hetta J. Problemas de sueño y síntomas de trastornos de la conducta alimentaria (TCA) en mujeres jóvenes. Eur J Psychiatry. 2004; 18(1):5-13.

10. Guía de Práctica Clínica para el Manejo de Pacientes con Insomnio en Atención Primaria. Ministerio de Sanidad y Política Social; 2009. Disponible en: http:// www.guiasalud.es/GPC/GPC_465_Insomnio_Lain_Entr _compl.pdf

11. Sarrais F, Castro Manglano P. El insomnio. An Sist Sanit Navar. 2007;30(Supl. 1):121-34.

12. Seguel LM. Trastornos del sueño en trastornos del ánimo y de la conducta alimentaria. Rev Med Clin Las Condes. 2013;24(3):487-93.

13. Sánchez J, Rosa Al, Olivares J. Las técnicas de relajación en el campo de la psicología clínica y de la salud en España: una revisión meta-analítica. Cuad Med Psicosom Psiquiatr Enlace. 1998;45-46:21-36.

14. Payne R. Técnicas de relajación. Guía práctica para el profesional de la salud. 5. ${ }^{\text {a }}$ ed. Barcelona: Paidotribo; 2005.

15. Martínez Escudero M, Cuellar Flores I, García-Consuegra Colado D, Pastorelli R, González Mateos V, Chinchurreta de Lora $\mathrm{N}$, et al. Taller de relajación en pacientes con trastorno de la conducta alimentaria. 
$14^{\circ}$ Congreso Virtual de Psiquiatria.com. Interpsiquis 2013.

16. Christian R, Ramos J, Susanibar C, Balarezo G. Risoterapia: un nuevo campo para los profesionales de la salud. Rev Soc Peru Med Intern. 2004;17(2):57-64.

17. Ruiz Gómez MC, Rojo Pascual C, Ferrer Pascual MA, Gimenéz Navascués L, Ballesteros García M. Terapias complementarias en los cuidados: humor y risoterapia. Index Enferm. 2005;14(48-49):37-41.

18. Calmet Torres L, Regalado Benitez M, Guevara Angulo M. Influencia de la risoterapia en las características psicológicas y sociales del niño escolar hospitalizado. Rev Enferm Hered. 2008;1(1):19-25.

19. Lomeli HA, Pérez Olmos I, Talero Gutiérrez C, Moreno CB, González Reyes R, Palacios L, et al. Escalas y cuestio- narios para evaluar el sueño: una revisión. Actas Esp Psiquiatr. 2008;36(1):50-9.

20. Walsh BT, Goetz R, Roose SP, Fingeroth S, Glassman AH. EEG-monitored sleep in anorexia nervosa and bulimia. Biol Psychiatr. 1985;20:947-56.

21. Delvenne V, Kerkhofs M, Appelboomfondu J, Lucas F, Mendlewicz J. Sleep polygraphic variables in anorexia nervosa and depression: a comparative study in adolescents. J Affect Disord. 1992;25:167-72.

22. Strauch I, Meier B. Sleep need in adolescents: a longitudinal approach. Sleep. 1988;11:378-86.

23. Maes M, Meltzer HY, Suy E, Minner B, Calabrese J, Cosyns P. Sleep disorders and anxiety as symptom profiles of sympathoadrenal system hyperactivity in major depression. J Affect Disord. 1993;27:197-207. 